\title{
Aprendiendo a investigar a través de la investigación-acción ${ }^{1}$
}

\section{Fraño Paukner Nogués}

orcid.org/oooo-0002-3460-3761

Universidad Católica del Maule, Chile

fpaukner@ucm.cl

\section{Rafael Sandoval Molina}

orcid.org/0ooo-0001-5443-5921 Universidad Católica del Maule, Chile rafael.sandoval@alu.ucm.cl

\section{Resumen}

Este artículo presenta los resultados de un estudio que comprometió la participación de alumnos chilenos en investigación-acción sistemática (I-A) como un medio para aprender a investigary mejorar sus competencias como estudiantes y futuros docentes. El estudio corresponde a un proyecto de investigación interno (PI 434196/2017) y fue realizado en un curso de pregrado (50 alumnos) correspondiente al segundo año de la carrera de Pedagogía en Inglés de la Universidad Católica del Maule, Talca, Chile. Los resultados revelaron que la investigación a través de la investigación-acción entrega a los alumnos la posibilidad de realizar procesos investigativos y reflexión critica acerca de su propia práctica. Los alumnos percibieron que la espiral autorreflexiva de reflexión y acción es crucial para establecer hábitos de búsqueda y reflexión. Los resultados sugieren que una falta de conocimiento previo acerca de investigación, por parte de los alumnos, afecta el crecimiento profesional y la preparación para el futuro desempeño docente; y muestran la necesidad de incluir la investigaciónacción sistemática en los programas de formación inicial docente, en Chile, para desarrollar hábitos de investigación y reflexión entre los futuros profesores.

Palabras clave (fuente: tesauro de la UnEsco). Ciencias de la educación; formación preparatoria de docentes; investigaciónacción; metodología; método de enseñanza.

1 El proyecto se denomina Aprendiendo a investigar a través de la Investigación-acción colaborativa, código PI 434196/2017. Proyecto interno patrocinado y financiado por la Universidad Católica del Maule, Talca, Chile y tuvo por finalidad vincular la investigación con la docencia de pregrado.

Recepción: 17/o6/2018 | Envío a pares: 11/08/2018 | Aceptación por pares: 21/08/2018 | Aprobación: 11/11/2018

DOI: 10.5294/edu.2018.21.3.7

Para citar este artículo / To reference this article / Para citar este artigo

Paukner-Nogués, F. y Sandoval-Molina, R. (2018). Aprendiendo a investigar a través de la investigación-acción. Educación y Educadores, 21(3), 504-519. DOI: 10.5294/edu.2018.21.3.7 


\title{
Learning How to Research through Action Research ${ }^{1}$
}

\begin{abstract}
This article presents the results of a study that involved Chilean students in systematic action research (AR) as a means to learn how to do research and improve their skills as students and future teachers. It was conducted in an undergraduate course with 50 second-year students of the English Teaching program at the Universidad Católica del Maule, Talca, Chile. The methodology used to obtain data was to carry out interviews, which were transcribed and subjected to an analysis using previous categories inherent in the questions and searching for emerging categories. The results revealed that research, through action research, gives students the possibility of carrying out research and critical reflection processes on their own practice. Students perceived that the self-reflexive spiral of reflection and action is critical to establish search and reasoning habits. It is concluded that students' lack of prior knowledge about research affects their professional growth and preparation for future teaching performance. It is necessary to include systematic action research in initial teacher training programs in Chile to develop research and reflection habits among future teachers.
\end{abstract}

\section{Keywords (Source: Unesco Thesaurus)}

Educational sciences; preservice teaching education; action-research; methodology; teaching methods. 


\section{Aprendendo a pesquisar por meio da investigação-ação}

\section{Resumo}

Este artigo apresenta os resultados de um estudo que contou com a participação de alunos chilenos na investigação-ação sistemática (I-A) como um meio para aprender a pesquisar e melhorar suas competências como estudantes e futuros professores. Foi realizado um curso de graduação com 50 alunos, correspondente ao segundo ano do curso de Pedagogia em Inglês da Universidade Católica de Maula, Talca, Chile. A metodologia utilizada para a obtenção dos dados consistiu na realização de entrevistas, as quais foram transcritas e submetidas a uma análise mediante o levantamento de categorias prévias próprias das perguntas e da busca de categorias emergentes. Os resultados revelaram que a pesquisa, por meio da investigação-ação entrega aos alunos a possibilidade de realizar processos investigativos e de reflexão crítica acerca de sua própria prática. Os alunos perceberam que a espiral autorreflexiva de reflexo e ação é crucial para estabelecer hábitos de busca e raciocínio. Conclui-se que uma falta de conhecimento prévio sobre a pesquisa, por parte dos alunos, afeta o crescimento profissional e a preparação para o futuro desempenho docente. Aponta-se a necessidade de incluir a investigação-ação sistemática nos programas de formação inicial docente, no Chile, para desenvolver hábitos de pesquisa e reflexão entre os futuros professores.

\section{Palavras-chave (Fonte: Tesauro da UnEsco)}

Ciências da Educação; formação preparatória de professores; pesquisa-ação; meto-dologia; método de ensino.

1 O projeto se denomina "Aprendendo a pesquisar por meio da Investigação-ação colaborativa”, código PI 434196/2017. Projeto interno patrocinado e financiado pela Universidade Católica de Maule, Talca, Chile, e teve como finalidade vincular a investigação com a docência de graduação. 
La necesidad de investigar y reflexionar para mejorar la práctica profesional aparece, en muchos estudios y propuestas, como un elemento a tomar en cuenta al momento de elaborar currículos de formación de profesionales, especialmente de profesores. En el mundo de hoy no sería posible formar profesores a la altura de los desafíos educacionales sin buscar, directa o indirectamente, capacidades para la investigación y la reflexión sobre la propia práctica. Un modelo que une ambas aspiraciones es la investigación-acción. Este paradigma obliga, gracias a sus ciclos recursivos de abordaje de los objetivos de investigación, a reflexionar acerca de lo que se hace mientras se hace.

Muchos paradigmas de desarrollo profesional para profesores en servicio todavía se organizan en torno a la noción tradicional del profesor como un mero técnico que sigue instrucciones. En el contexto chileno, en el cual este estudio fue realizado, las universidades todavía confían en paradigmas tradicionales de formación profesional. Sin embargo, esta clase de desarrollo profesional no promueve la práctica reflexiva y la investigación entre los profesores, y esta es solo un medio de transmitir conocimientos y habilidades a los profesores. Este estudio muestra la necesidad de mirar atentamente cómo se puede empoderar a futuros profesores chilenos para llegar a ser prácticos y reflexivos, comprometiéndolos en investigación-acción colaborativa. El estudio también enfatiza la importancia crítica de introducir la investigación-acción sistemática en los programas de formación docente con el objeto de promover hábitos de reflexión y búsqueda que los futuros profesores puedan luego utilizar en su práctica pedagógica.

\section{Marco teórico}

\section{Acción reflexiva e investigación-acción}

El concepto de reflexión es muy antiguo, pero su uso en el ámbito educacional no lo es tanto. El primero en proponerlo fue Dewey (1933), pero fue
Schön quien insistió en las nociones de reflexión en la acción y sobre la acción, para demostrar que los profesores no son técnicos, esto es, aplicadores de cosas pensadas y diseñadas por otros, llamados expertos (Aulls y Shore, 2008; Gore y Zeichner, 1991; Leitch y Day, 2000). La reflexión concebida por Schön va más allá de lo meramente cognitivo, propuesto por Dewey, y a punta a la relación entre acción y pensamiento, esto es, a pensar acerca de lo que se hace mientras se hace (Ferraro, 2000, Leitch y Day, 2000; Paukner, 2005; Van Manen, 1995; Bing, Blondeau, Dreher e Irby, 2015).

La reflexión en el trabajo pedagógico supone que los docentes no solo debieran pensar mientras realizan acciones (reflexión en la acción), sino que también debieran realizar una reflexión más profunda acerca de sus acciones y el impacto que estas tienen en el aprendizaje de sus alumnos (reflexión sobre la acción) (Van Manen, 1995). Este concepto ha sido llamado T2 in action: teaching and thinking in action o T2 (teaching \& thinking) (Bing et al., 2015). Por otra parte, la reflexión de los profesores en y sobre sus prácticas debiera afectar sus actitudes, sus ideas y creencias, su identidad y su rol como agentes de cambio (Gore y Zeichner, 1991; Leitch y Day, 2000; McMahon, 1999).

\section{Reflexión sobre la práctica e investigación-acción}

La práctica reflexiva -en que se combinan reflexión en y sobre la práctica- se concibe como un medio para que los profesores -en formación y en servicio- mejoren sus capacidades profesionales y, por lo tanto, su desempeño profesional (Feldman, Altrichter, Posch, y Somekh, 2018). La práctica reflexiva, entonces, aparece como una "habilidad blanda" que determina, en principio, el éxito pedagógico futuro (Ashcroft y Foreman, 1994; Aulls y Shore, 2008; Ferraro, 2000; McMahon, 1999; Riel, 2017).

Reflexión sobre la práctica e investigaciónacción son conceptos cercanos que, muy a menudo, se confunden e incluso se usan indistintamente 
(Johnston, 2005; McMahon, 1999). Ambos suponen mejorar la práctica, optimizar el desempeño profesional y mejorar el aprendizaje y la motivación de los estudiantes. Empero, la investigación-acción es un método, una forma planificada de enfrentar un desafío y de solucionar un problema (McMahon, 1999). Aquellos profesores que realizan procesos de práctica reflexiva no necesariamente lo hacen a través de la investigación-acción. Además, la práctica profesional mejora, si se concibe no solo como la adquisición de competencias para la práctica y para la investigación, sino también como desarrollo de habilidades reflexivas y críticas (Feldman et al., 2018; Mann y Walsh, 2017). La profesión docente en el mundo de hoy exige poseer saberes, pero también construir saberes fundados en la experiencia por medio de la práctica reflexiva (Mata, Hernández y Centeno, 2018).

\section{Modelos de investigación-acción}

La espiral de ciclos, propuesta por primera vez por Lewin en 1952 y revisada por Elliot en 1991 (citado en Leitch y Day, 2000), contempla ciclos de reflexión y acción tales como planificar, actuar, recolectar evidencia, reflexionar y realizar nuevas acciones. Cada ciclo entrega entendimiento más profundo, el cual guía el modo de mejorar las acciones (Riel, 2017). Siguiendo la tipología de Grundy (citado en Leitch y Day, 2000), que diferencia entre modelos técnicos, prácticos y emancipatorios de I-A, la espiral de autorreflexión correspondería más al modelo práctico. La I-A práctica se identifica con el modelo de profesor reflexivo propuesto por Ashcroft y Foreman (1994), debido a que enfatiza el involucramiento de los profesores en una búsqueda más sistemática que aquella implicada en la noción de práctico reflexivo, propuesta por primera vez por Schön (McMahon, 1999). Sin embargo, algunos han argüido que la habilidad de la I-A práctica para producir cambios en los profesores es limitada (Leitch y Day, 2000; Van Manen, 1995). Según Leitch y Day (2000) y que es necesario un modelo de I-A más emancipatorio, para producir cambios no solo en el ámbito individual, sino también en los espacios sociales y educacionales. El modelo emancipatorio insiste en una autoespiral de reflexión y acción, que se focaliza no solo en el mejoramiento de la práctica de los profesores, sus actitudes y sus teorías personales, sino también en los cambios que deben realizarse en los sistemas educacionales y sociales (Gore y Zeichner, 1991; Leitch y Day, 2000; Van Manen, 1995).

\section{Investigación-acción colaborativa}

En un comienzo, la intención de la investigación-acción fue comprometer, individualmente, a los profesores en un proceso de reflexión y búsqueda. Incluso los trabajos de Whitehead -más en consonancia con el enfoque emancipatorio- se focalizan en el proceso de introspección individual, más que en el colectivo (Leitch y Day, 2000). La importancia de tener a los profesores conectados, hablando sobre sus propias experiencias en el aula y creciendo profesionalmente juntos ha ganado terreno durante las últimas décadas. El diálogo juega también un rol esencial en el proceso de la investigación-acción colaborativa. Por lo tanto, más que focalizarse en que el profesor, individualmente, realice I-A en el aislamiento de su propia aula, la investigación-acción colaborativa enfatiza la colaboración entre profesores e incluso entre compañeros universitarios por medio de la "apertura de un espacio comunicativo" (Kemmis y McTaggart, 2005, p. 578).

En la investigación-acción colaborativa, los miembros del grupo se comprometen en un diálogo colaborativo con el objeto de examinar detenidamente sus prácticas pedagógicas habituales y los supuestos y creencias que subyacen a las estrategias pedagógicas que tienen a su disposición. De este modo surge una suerte de comunidad que se orienta a construir conocimiento (Muñoz y Garay, 2018). A través de sus acciones comunicativas -o en la esfera pública, como sugiere Habermas (1989)-, los profesores se involucran en una reflexión más profunda acerca de tópicos que trascienden las cuatro paredes de su aula. La investigación-acción co- 
laborativa permite al grupo reflexionar acerca de y buscar en asuntos políticos y sociales que afectan sus contextos educacionales. Este proceso, empero, se da progresivamente y es necesario considerar tiempos para la adquisición de herramientas, procedimientos y estrategias (Mata et al., 2018).

\section{La investigación-acción en América Latina}

En América Latina, la I-A apareció por primera vez alrededor de 1960 como un movimiento político que combinó la educación popular, la Teología de la Liberación, la comunicación alternativa, la Filosofía de la Liberación y la investigación-acción participativa (IAP). El principal objetivo de la I-A fue permitir a las personas deprivadas entender su propia realidad, para cambiarla. Hoy, este movimiento es conocido como el paradigma de la emancipación, un enfoque que combina la reflexión y la acción y se basa fuertemente en el profundo conocimiento que las personas tienen de sus propios contextos, y que les permite trabajar a partir de ellos. Algunos de los que adoptan este enfoque aplican las ideas de Paulo Freire para encontrar soluciones para un amplio rango de problemas sociales (incluida la educación) a través de la reflexión y de la práctica. En Colombia, el sociólogo Orlando Fals Borda expresamente destacó la "acción" como elemento fundamental de la investigación acción participativa (Ortiz y Borjas, 2008). Dado que las ideas de Fals Borda combinan pensamiento con acción, entendimiento con cambio y saber con hacer, ellas han sido una inspiración para educadores y otros que necesiten llevar las ciencias sociales a la práctica. Fals Borda incluso se anticipa a Michel Foucault. En sus estudios acerca de los campesinos en la Costa Atlántica de Colombia, perfila el concepto de discurso como producción de poder (Martín, 2014).

El profesional y, en especial, el profesor, como investigador -a nivel escolar y universitario- no está claramente presente en América Latina. Eso ha dificultado tanto la inserción laboral como la práctica profesional y ha impedido al profesor con- formar comunidades y redes de producción de conocimiento y reflexión sobre la práctica. He aquí un gran desafío para la formación inicial docente (Muñoz y Garay, 2018).

La tradición de la I-A no está bien establecida en Chile. Según el Marco para la Buena Enseñanza (2008) del Ministerio de Educación, la reflexión sistemática acerca de la propia práctica es parte de las responsabilidades profesionales del profesor. Sin embargo, esta capacidad no es parte del currículo formal de la mayoría de los programas de formación docente, y la mayoría de los profesores chilenos deben enfrentar su trabajo de aula sin haber sido previamente formados en la reflexión sistemática y en la práctica de la investigación.

\section{Propósito del estudio}

Aunque un gran cuerpo de investigación entrega evidencias acerca del potencial de la investigación-acción y de la práctica reflexiva para promover el desarrollo profesional entre los profesores, la mayoría de estas investigaciones se dan en los contextos de Norteamérica, Europa y Australia, en donde se han desarrollado las nociones de práctica reflexiva e investigación-acción. El propósito de este estudio fue involucrar alumnos de pregrado en investigación-acción como una forma de fundamentar el concepto de práctica reflexiva crítica e investigación como parte de sus propias actitudes, creencias e identidad profesional, y promover la noción de los profesores como productores de conocimiento.

\section{Metodología}

\section{Diseño}

La investigación-acción colaborativa utilizada para este estudio fue inspirada por el proceso sistemático y cíclico propuesto en la espiral de la investigación-acción, lo que supone los siguientes pasos: planificación, recolección de datos, análisis y presentación de los datos y planificación nuevamente. Primero, fue presentado un proyecto de investigación 
que, al mismo tiempo, solicitó los fondos y los permisos. Segundo, se eligió el curso de Educación Comparada, durante el primer semestre de 2017, segundo año de la carrera de Pedagogía en Inglés. Objetivo general: involucrar a los estudiantes de un curso de pregrado en un proceso de levantamiento de competencias investigativas, reflexivas y de trabajo en equipo por medio de la investigación-acción colaborativa. Objetivos específicos: lograr en los estudiantes participantes competencias: 1) para investigar a través de la investigación-acción colaborativa; 2) para reflexionar acerca de la propia práctica; 3) para trabajar en equipo.

Primer ciclo: En la primera clase, en marzo de 2017, los alumnos fueron informados de la modalidad del curso mediante la presentación del proyecto de investigación adjudicado. El objetivo de esta presentación fue realizar una introducción a la espiral de reflexión y acción que caracteriza la I-A (Kemmis y McTaggart, 2005; Riel, 2017). Posteriormente, el curso fue dividido en seis grupos y a cada grupo se le asignó una zona geográfica (Europa, África, Oceanía, Asia, América del Norte y América del Sur). Cada grupo debía elegir un país representante de cada zona geográfica para compararlo con Chile. En ese momento se hizo un sorteo para determinar el orden de las presentaciones.

Durante las tres o cuatro clases siguientes, los alumnos fueron introducidos tanto a la educación comparada como a los fundamentos de la investigación-acción. Luego se estableció un calendario de presentaciones para los resultados de la primera vuelta. Durante los meses de abril y mayo, los grupos hicieron sus presentaciones. Cada una fue retroalimentada por el curso en pleno para su evaluación y corrección en el segundo ciclo. Inmediatamente después de su presentación, cada grupo fue entrevistado acerca de la investigación asociada a la preparación de la presentación. Las entrevistas siguieron dos grandes líneas: en primer lugar, conseguir información acerca de los detalles técnicos de la investigación (acceso a la información, búsqueda, fuentes, etc.). En segundo lugar, conseguir información acerca de la experiencia de investigar en equipo (dividir el trabajo, asignar roles, colaborar, etc.)

SEGUndo Ciclo: La segunda ronda de presentaciones tuvo lugar entre los meses de junio y julio. Al igual que en el primer ciclo, después de cada presentación tuvo lugar una retroalimentación y evaluación general con miras a la entrega del informe final. Cada grupo fue entrevistado de acuerdo con las mismas categorías del ciclo anterior. En esta segunda entrevista, empero, se trató de conseguir información acerca de los posibles aportes de la actividad para la formación inicial de los estudiantes.

\section{Participantes}

Participaron cincuenta estudiantes, esto es, el segundo año de la carrera de Pedagogía en Inglés de la Universidad Católica del Maule, Talca, Chile. Dichos estudiantes no tenían formación alguna en investigación (los cursos de metodología de la investigación se imparten hacia el final de la carrera). El proyecto también involucró al profesor del curso de Educación Comparada, el cual se había adjudicado previamente el proyecto de investigación, y a un ayudante de investigación, alumno del mismo curso.

\section{Recolección y análisis de datos}

Los datos recolectados para este proyecto provienen de las diez entrevistas realizadas a cinco grupos de alumnos. Una después de cada ciclo de investigación. Cada entrevista duró entre veinte y treinta minutos. Las entrevistas fueron transcritas por el asistente de investigación y revisadas por el investigador. Para el análisis de los datos se usó un sistema de codificación propio de los diseños de investigación cualitativos. El análisis de los datos iniciales se obtuvo buscando categorías emergentes iniciales surgidas de las entrevistas, categorías iniciales que a su vez surgieron de los temas presentes en las preguntas de las entrevistas y fueron guiadas por el marco conceptual del estudio. Un segundo 
set de categorías surgió desde las respuestas de los estudiantes durante las entrevistas, que fueron calificadas como subcategorías emergentes. Cada categoría es presentada según la interpretación de los investigadores, seguida en algunos casos por citas de las transcripciones para apoyar la evidencia.

\section{Resultados}

\section{Categorias emergentes iniciales}

1. EVALUACIÓN GeNeRAL DE LA EXPERIENCIA DE INVESTIGAR: Esta categoría surgió como la principal en todos los grupos y aparece, en general, con una connotación muy positiva. Los alumnos valoraron el haber podido realizar una investigación fruto de un proyecto, lo que aseguraba su seriedad, y disfrutaron el conocer cosas nuevas a través del proceso investigativo. Las opiniones, en general, señalan que la experiencia de investigar en forma acotada y disciplinada es de gran valor intelectual, profesional y moral, dado que las competencias investigativas trascienden el hecho puntual y se constituyen en capacidades útiles en otro ámbitos: la disciplina, el orden, la perseverancia, la capacidad de "separar la paja del trigo" y la capacidad de indagación constituyen atributos necesarios para insertarse adecuadamente en la sociedad del siglo XXI. En otras palabras, en "competencias blandas" o capacidades para la vida. Por otra parte, las opiniones también insisten en las dificultades que conllevó la investigación, aunque se perciben legitimadas por la magnitud de la tarea. Consultado sobre cómo evaluaba la experiencia general, un alumno dijo: "Desafío útil y complicado. Divertida. Aprendimos qué es investigar". Otro planteó: "Se hizo difícil en algunos aspectos, en especial en obtener información actualizada de las áreas que buscábamos".

2. IMPORTANCIA DEL INGLÉS PARA INVESTIGAR: LOS alumnOS, en general, manifestaron que, dado que sus investigaciones fueron realizadas a través de Internet y eran acerca de sistemas educacionales extranjeros, el dominio del idioma inglés fue relevante para ob- tener información en sitios web. Las opiniones insisten en la ventaja que representa el dominio del idioma inglés para la investigación, de lo cual se desprende este rasgo como una importante competencia profesional que debiera ser parte de la formación inicial docente. Todo profesional debería saber inglés, especialmente aquellos que van a investigar. Consultado sobre la importancia del inglés para investigar, un alumno manifestó: "Muy importante. Casi toda la información de nuestro tema estaba en inglés. Si no hubiera sabido inglés, no habría podido obtener casi nada".

Dado que los alumnos participantes en este proyecto estudiaban Pedagogía en Inglés, contaban con el suficiente dominio del idioma como para utilizar la información de internet que encontraron en inglés, lo que les permitió realizar el trabajo mientras reparaban en la ventaja que significaba saber inglés. Esto implicó, entonces, una efectiva reflexión sobre la práctica, esto es, acerca de lo que se hace mientras se hace. Como rasgo profesional, podemos relevar esta condición, si le damos una connotación afectiva insistiendo en la eventual satisfacción profesional del quehacer bien hecho. En otras palabras, un profesional que está realizando bien sus funciones gracias a la puesta en operación de sus competencias profesionales debería sentir satisfacción y plenitud.

Sería interesante conocer qué podría ocurrir si los investigadores no dominaran el idioma inglés. Tal vez en ese caso solo aparecería la frustración y la deseabilidad:una especie de reflexión negativa, esto es, reflexionar acerca de lo que no se está haciendo por falta de capacidades.

3. DISPONIBILIDAD DE LA INFORMACIÓN: LOS alumnos coinciden en que hay mucha información disponible, que hay que saber procesar, para distinguir lo verdadero de lo falso. "Separar la paja del trigo" aparece como un elemento fundamental al buscar información, especialmente al usar internet. Las opiniones manifestadas coinciden en destacar la inmensa cantidad de información disponible en internet, junto con una inmensa cantidad de desin- 
formación. Resulta una proeza distinguir lo que vale la pena de lo que no. Esta aparente dificultad entraña, empero, una ventaja: la necesidad de desarrollar capacidades analíticas (observación, comparación, contrastación) para distinguir tipos de información y optar por la más relevante. Un alumno manifestó: "La información está disponible, pero hay que ser cuidadoso al procesarla".

4. UsO DE FUENTES: LOS alumnos manifestaron que los datos fueron obtenidos de entrevistas, artículos y, principalmente, de internet. A propósito del uso de internet, los alumnos insistieron en lo novedoso que significó realizar búsquedas profundas y con sentido en sitios web de instituciones como embajadas, escuelas y universidades en el extranjero. Su experiencia en internet se reducía al uso de redes y a la búsqueda de información no muy relevante. Las opiniones apuntan a destacar que, en principio, todo es fuente de información, porque esta puede estar en los lugares más insospechados. Un alumno expresó: "La información disponible es infinita. El problema está en encontrarla y seleccionarla".

La rutina de navegación de los alumnos los hace desconocer el potencial informativo de sitios que solo son descubiertos y revelados cuando hay una búsqueda acotada en función de metas y objetivos. Eso implica, entonces, la posesión de criterios de búsqueda. La experiencia de investigar permitió a los estudiantes reflexionar con respecto a la calidad de las fuentes y desarrollar criterios de búsqueda y selección de información, así como la extrapolación de dicha reflexión y dichos criterios a otros ámbitos de su quehacer. Un estudiante aseveró: "Ahora, si no está demostrado por algo, una institución así con experiencia, con recorrido, yo no le creo. Y eso aplica también para la vida diaria, para la vida personal de cada uno. Yo lo trato de llevar a otros aspectos". Otro alumno planteó: "Se fue más preciso y exhaustivo en la investigación, se filtró mejor lo que era relevante para la investigación, y se hizo más análisis e interpretación de la misma".
5. Ganancia personal: Los alumnos manifestaron que el proceso de investigación significó para ellos crecimiento profesional y personal. El análisis arrojó cuatro subcategorías:

Ganancia profesional: Esta subcategoría surgió de afirmaciones acerca de lo importante que resulta aprender a investigar para una buena formación profesional, así como para el futuro desempeño profesional. Los alumnos plantean como valioso el aprender a buscar y procesar información, el saber distinguir la información relevante de la superflua, pero, sobre todo, el haber despertado a la verdad acerca de la presencia de fuentes no confiables. Uno de los estudiantes reflexionó: "Yo creo que es importante para los profesores tener las dotes para investigación, así pueden estar actualizándose constantemente. Hay investigaciones que refutan ideas que en el pasado se creían que eran correctas, pero que últimamente se han desmentido. Entonces, es importante estar actualizándose en lo que sucede respecto a la educación". Y agregó: "Al investigar, uno desarrolla varias habilidades al mismo tiempo, porque uno tiene que discernir de la información que es correcta de la incorrecta, saber resumir la información, encontrar los puntos clave, qué es lo que uno pretende informar a los demás. Entonces, sin ese tipo de habilidades el profesor se puede quedar algo estancado".

Ganancia cultural: Esta subcategoría surgió de la experiencia de investigar acerca de otros continentes, otros países y otros sistemas educacionales. Los alumnos manifestaron que fue una enriquecedora experiencia el leer acerca de otras culturas y lograr, directa e indirectamente, conocimientos acerca del mundo. Una alumna lo expresó así: "La investigación fue un viaje. Para nosotras, viajamos a Finlandia, fuimos a Europa y volvimos, pero sin haber viajado. Al final la investigación, internet, las fuentes, nos ayudan a eso, a viajar sin viajar". Otro estudiante aseveró al respecto: "Uno al saber de más países va encontrando patrones que hacen que tal cosa mejore el país y esos patrones tal vez se pueden a repetir en Chile". 
Ganancia ética: Esta categoría surge de haber tenido que enfrentar el desafío de la honestidad a la hora de seleccionar información y enfrentar el reto de basar las conclusiones en evidencias. Los alumnos manifestaron lo importante que es lograr cadenas de información correcta. Un alumno manifestó: "Saber cerciorarse de la información es vital para quien llegue a ser profesor, para no pasar información incorrecta a sus alumnos".

Ganancia académica: Esta subcategoría surge de la reflexión que los estudiantes hicieron de su propio proceso de investigación. Expresaron que gracias a esta experiencia pudieron mejorar su actitud hacia la adquisición de conocimiento: les permitió detectar que estaban dedicando demasiado tiempo a asuntos poco importantes, fomentó su capacidad de análisis, y aumentó su aprecio por información que antes consideraban irrelevante o no interesante. Una estudiante afirmó: “Lo que a mí me gustó más, porque es algo que yo siempre había evitado, fue enfrentarme a los gráficos y a los datos, y ocuparlos en una argumentación".

6. LA EXPERIENCIA DE INVESTIGAR COLABORATIVAMENTE: LOS alumnos coinciden en lo positiva que fue la experiencia de investigar colaborativamente, aunque insisten en lo difícil que resultó, en un comienzo, ponerse de acuerdo y coordinarse para enfrentar las tareas. Los alumnos plantean que aprendieron a respetarse, a comunicarse, a escuchar las opiniones de los otros, pero luego de un proceso de ajuste no exento de problemas. Un alumno dijo: "Al principio no se tomó el trabajo en serio, quizás por no entender la magnitud del mismo. Luego se aceleró el trabajo, y nos dimos cuenta de lo que podíamos hacer si es que de verdad le poníamos ganas al asunto".

\section{Categorias emergentes secundarias}

1. El APRENDIZAJE COMO UNA CUESTIÓN DE DECISIÓN PERSONAL: Los alumnos manifestaron que, definitivamente, investigar es una poderosa forma de aprender que depende de la decisión de cada uno. Hay tanta infor- mación a disposición de todos, gratuitamente, que no hay excusa para no aprovecharla en beneficio propio. Un alumno manifestó: "Uno puede hacerse experto en prácticamente cualquier tema al tener habilidades de investigación".

2. El desarRollo de EXPERIENCIAS DE INVESTIGACIÓN EN EL CURRÍCULO DE LA FORMACIÓN INICIAL DOCENTE (FID): LOS alumnOS percibieron que la realización de investigaciones y, por ende, el desarrollo de capacidades investigativas refuerzan la FID. Un alumno lo planteó así: "Enseñar competencias investigativas a estudiantes de primer año contribuiría a que aprendieran mucho más y egresaran mucho mejor preparados".

3. LA IMPORTANCIA DE LA LABOR DE UN LÍDER: LOS alumnOS percibieron que para llevar adelante un proyecto de investigación colaborativa es fundamental la figura de un buen líder que aglutine y organice el trabajo. Un alumno lo planteó en los siguientes términos: "El trabajo en equipo fue más eficiente gracias al líder de grupo, que orientó recursos y esfuerzos de modo que los maximizó. No hizo el trabajo más fácil, pero sí más eficiente". También advirtieron el riesgo de descansar en esa persona demasiada responsabilidad y de que el resto del grupo se desentienda de la suya. En uno de los grupos, que no eligió a un líder, una de las estudiantes asumió ese rol espontáneamente. Un miembro de dicho grupo declaró que "sería ideal no tener como un jefe que cargue toda la responsabilidad, porque el trabajo es en conjunto, en grupo", y añadió que "todos deberían tener la misma carga, para que sea más equitativo".

\section{Interpretación y argumentación de los resultados}

Las categorías halladas, presentes en muchos estudios revisados, representan, empero, algo nuevo en el contexto de la formación inicial docente chilena. La investigación, en general, y la I-A, en particular, están prácticamente ausentes de los currículos de formación de profesores en las universidades. Los resultados confirman la necesidad de incorporarlos 
en función de la necesidad que suponen y de la buena disposición de los participantes a incursionar en actividades de I-A. Las categorías encontradas en el análisis de los resultados, desde el punto de vista de su aporte a la discusión científica, se pueden dividir en tres grandes ejes:

1. La valoración que hacen los estudiantes de la oportunidad de aprender a investigar nos lleva a considerar que, al parecer, se percibe la necesidad como una deficiencia a la hora de enfrentar una carrera profesional, especialmente pedagógica. Por lo tanto, emerge la percepción de la necesidad de incorporar los cursos de metodología de la investigación, con sus correspondientes pasos prácticos, al comienzo de la carrera y no hacia el final, como suele hacerse.

2. Los elementos de la dinámica social (de trabajo en equipo, de liderazgo, de roles), propios de la investigación-acción colaborativa, surgen también como una necesidad de la formación profesional que debe buscarse directamente, a través del currículo, e indirectamente, a través de acciones que propicien el trabajo en equipo y, por sobre todo, el trabajo en redes. En este contexto emerge la necesidad de considerar el desarrollo de dichas habilidades como propedéuticas a la carrera profesional docente, a nivel de la educación secundaria.

3. La posibilidad de investigar, especialmente usando internet, como una decisión personal nos lleva a la consideración de estimular la curiosidad y el deseo de llegar más allá de nuestros alumnos. Habiendo tanto material para aprender y buscar la excelencia como profesional y como persona, todos, en principio, podrían optar por ello. “¿Por qué no lo hacen?" parece surgir como pregunta obvia. La respuesta, con base en nuestros hallazgos, es compleja. Por una parte, porque no saben lo importante que es. Solo al tener la experiencia descubren cuánto lo necesitan y lo fundamental que es para su formación profesional y humana. Por otra parte, el hecho de que esta información sea, al menos aparentemente, de tan fácil acceso hace que no exista la curiosidad o el deseo de adquirirla como conoci- miento propio. La información y los datos se están almacenando en la nube, como si se tratara de un disco duro externo, y no en el cerebro, como hacían las generaciones previas a internet. Esto podría llevar a los alumnos a confiar en que la información siempre está ahí, disponible, y a pensar que pueden recurrir a ella cuando es necesario.

\section{Conclusiones}

El estudio muestra que la vasta literatura tanto acerca de la práctica reflexiva como acerca de la investigación-acción ha sido asertiva en cuanto a mostrar las capacidades de indagar y reflexionar como inherentes a la condición profesional, ya sea en formación o en ejercicio. En este caso particular fue posible unificar ambos conceptos en un proceso de inducción a la investigación a través de la investigación-acción, con el objeto de promover la reflexión acerca de la propia práctica, basado en el supuesto de que la investigación debería ser una tarea frecuente en el desempeño profesional. Los resultados de esta investigación muestran la mejora que significa, para estas deficiencias, un proceso de investigación como parte de la formación profesional, lo que implica mostrar dichas deficiencias con la práctica misma, como ejercicio de metacognición a partir del desempeño profesional, de profesionales en formación, pero profesionales.

Los alumnos involucrados en el estudio aprendieron a investigar siguiendo un método definido, acotado en el tiempo y focalizado en los temas a investigar, mientras descubrían la necesidad de trabajar en equipo, organizarse en torno a un líder, conocer el idioma inglés y desarrollar el gusto por la investigación. La tarea investigativa, por ser un modelo de I-A, supuso la adquisición de competencias más allá de lo investigativo que son útiles en el desempeño profesional en general (trabajo en equipo, formación de redes, liderazgo, roles).

Si concluimos en función de los objetivos de la investigación, los alumnos, al involucrarse por primera vez en un proyecto formal de investigación, 
desarrollaron competencias para investigar en un proceso recursivo de trabajo colaborativo. Eso implicó aprender a descubrir fuentes, destacar la información relevante, dividir el trabajo y asignar tareas, planificar un segundo ciclo en función de la retroalimentación del primero y, sobre todo, trabajar en equipo. Los alumnos descubrieron la importancia de la investigación planificada y sistemática como garantía de certeza de la calidad de la información y, por tanto, de validación de datos para la toma de decisiones. Esto fue percibido como un importante elemento de la formación profesional, en general, y de la formación inicial docente, en particular (Feldman et al., 2018).

Durante el proceso de investigación y debido al diseño, así como a elementos emergentes, los alumnos tuvieron que reflexionar acerca de su quehacer. Enfrentados a una mayor cantidad de información de calidad en inglés que en castellano, apreciaron su propia ventaja comparativa de ser personas con conocimientos de esa lengua. Así, reflexionaron sobre la acción durante la acción y derivaron de la experiencia una emoción positiva hacia sus propias competencias y el efecto que tienen en su desempeño profesional. La reflexión acerca de la propia práctica aparece como elemento de la formación profesional que contienen los textos y suele enseñarse como parte de cursos teóricos. Los resultados de esta investigación muestran lo profundo y enriquecedor que resulta enseñar esta capacidad in situ, como parte del proceso mismo, lo que permite la percepción inmediata del aporte a la formación profesional.

Del mismo modo, el descubrimiento de fuentes confiables y el discernimiento desarrollado para distinguir la calidad de las fuentes fueron hechos que no escaparon a su percepción y reflexión durante el proceso. Las conclusiones y actitudes derivadas de la reflexión fueron llevadas a la práctica en la segunda parte de la investigación y en la segunda exposición, lo que permitió que la reflexión sobre la acción durante la acción influyera en la acción.
Se puede afirmar, por tanto, que el estudio arroja resultados que coinciden con las investigaciones previas y la literatura sobre los modelos de investigación-acción propuestos y su eficiencia para fomentar el desarrollo de competencias para reflexionar sobre su propio desempeño profesional. Empero, los resultados de esta investigación muestran que esto se puede llevar a cabo en ambientes de investigación-acción colaborativa, con lo cual se logran ganancias adicionales que van más allá de lo investigativo y reflexivo y que impactan la formación profesional en desarrollo y que debieran tener impacto en el ejercicio profesional.

Los estudiantes que fueron parte de este estudio se enfrentaron a la necesidad de trabajar colaborativamente, identificaron los problemas derivados de esta forma de trabajo y buscaron formas de solucionarlos. Captaron la necesidad de coordinarse, de respetar las opiniones de los demás, de establecer tiempos de trabajo y de cumplir con ellos.

Además, se hizo patente para ellos la necesidad de la existencia de un líder o coordinador general, que tomara algunas decisiones necesarias que no se hubiesen tomado previamente en grupo y que velara por la unidad y coherencia de la investigación. Algunos de ellos reflexionaron sobre la importancia de que las labores individuales estuvieran bien definidas, incluyendo las del líder, para no recargar a nadie de trabajo ni afectar -entre otras cosas- su buen resultado. Nos parece fundamental destacar la importancia del liderazgo de equipos de investigación, tema bastante ausente en la literatura revisada. La investigación mostró la importancia de guiar adecuadamente el proceso de I-A para el logro de los objetivos y para la organización humana que esto supone. Pensamos que un proceso investigativo de esta naturaleza, sin el adecuado liderazgo, podría terminar en el fracaso de la investigación $y$, por ende, en un rechazo a la actividad investigativa por parte de los profesionales en formación.

Este proceso de investigación, en vista de lo anterior, logró que los estudiantes participantes desa- 
rrollaran competencias para investigar a través de la investigación-acción colaborativa, para reflexionar sobre su propia práctica y trabajar en equipo. Para que estas capacidades se desarrollen, deben ser enseñadas a través de procesos formales y, en lo posible, ser incluidas en el currículo (Mann y Walsh, 2017). De esto surgió también la percepción de la necesidad de desarrollar estas capacidades como parte de cualquier currículo de formación profesional, especialmente en el caso de las carreras de Pedagogía. En el mundo virtualizado, tecnologizado, globalizado y organizado en redes, se hace fundamental desarrollar la capacidad de investigar para conseguir información relevante y descartar aquella que no lo es, especialmente la concebida con el ánimo de desinformar. El fenómeno de la posverdad, caro a las redes sociales, interpela a las personas, especialmente a los formadores, a desarrollar capacidades para lidiar con ella.

Por último, además de desarrollar algunas competencias de investigación, muchos de los estudiantes participantes de este proyecto también desarrollaron el deseo de investigar. Podría decirse que la I-A despertó su curiosidad y les devolvió una sensación o actitud positiva hacia la adquisición de conocimientos, incluso venciendo la idea de la pérdida de curiosidad por la disponibilidad de la información. En otras palabras, puede afirmarse que usar la I-A como método de pudiera vencer la apatía hacia la investigación y, en general, hacia el aprendizaje.

\section{Limitaciones de la investigación y sugerencias para futuros estudios}

Esta investigación fue realizada en condiciones limitadas, con un diseño acotado y con falta de experiencia por parte de los alumnos. Eso significó que la extensión y la profundidad de los ciclos resultaran insuficientes para apuntar a logros más profundos y permanentes en cuanto al desarrollo de nuevas categorías de análisis y hábitos de desempeño profesional. En otras palabras, la experiencia no caló lo suficientemente hondo en los estudiantes como para hablar de un nuevo rasgo profesional. Para lograr esto se necesitaría una línea transversal al currículo de formación inicial docente que permitiera reforzar las conductas, hasta afianzarlas. Si bien el proceso implicó solo ganancias para los estudiantes, estas pudieron haber sido superiores y permanentes, de haberse dado en condiciones más estables. Un curso universitario de un semestre de duración no permite logros de gran envergadura.

Estudios venideros acerca de esta temática podrían ser un aporte, si apuntan al logro de hábitos de investigación buscando nuevas temáticas y nuevos diseños que permitan un trabajo más a largo plazo y en condiciones más globales. También sería deseable que los estudiantes recibieran algún apresto o propedéutica, antes de embarcarse en un proyecto de investigación.

\section{Referencias}

Ashcroft, K.y Foreman-Peck, L. (1994). Managing teaching and learning in further and higher education. Londres: Falmer Press.

Aulls, M. W. y Shore, B. M. (2008). Inquiry in education: Vol. 1. The conceptual foundations for research as a curricular imperative. Nueva York: Taylor \& Francis.

Badia, G. (2017). Combining Critical Reflection and Action Research to Improve Pedagogy. Libraries and the Academy, 17(4), 695-720. DOI: 10.1353/pla.2017.0042

Baskerville, R.y Myers, M. D. (2004). Foreword. MIS Quarterly, 28, 329-335. 
Bing-You, R. G., Blondeau, W., Dreher, G. K. e Irby, D. M. (2015). T2 (teaching \& thinking)-in-action skills of highly rated medical teachers: How do we help faculty attain that expertise? Innovations in Education and Teaching International, 54(5), 409-417. DOI: 10.1080/14703297.2015.1093425

Burbank, M. D. y Kauchak, D. (2003). An alternative model for professional development: Investigations into effective collaboration. Teaching and Teacher Education, 19, 499-514. 10.1016/S0742-051X(03)00048-9

Cruickshank, D. R. (1987). Reflective teaching. Reston: Association of Teacher Educators.

Denos, C., Toohey, K., Neilson, D.y Waterstone, B. (2009). Collaborative research in multilingual classrooms. Salisbury: MPG Books.

Dewey, J. (1933). How we think: A restatement of the relation of reflective thinking to the educative process. Chicago: Henry Regnery.

Dolbec, A. y Clément, J. (2000). La recherche-action. En T. Karsenti y L. Savoie-Zajc (eds.), Introduction à la recherche en éducation (pp. 199-224). Sherbrooke: CRP.

Domingo, Á. (2009) ¿Qué és la práctica reflexiva? Butlletí d’Edu21, 22.

Feldman, A., Altrichter, H., Posch, P. y Somekh, B. (2018). Teachers investigate their work. An introduction to action research across the professions. Nueva York: Routledge

Ferraro, J. M. (2000). Reflective practice and professional development. Washington DC: U.S. Department of Education.

Glaser, B. G.y Strauss, A. L. (1967). The discovery of grounded theory: Strategies for qualitative research. Cambridge: Cambridge University Press.

Gore, J. M. y Zeichner, K. M. (1991). AR and reflective teaching in pre-service teacher education: A case study from the United States. Teaching and Teacher Education, 7, 119-136. DOI: 10.1016/0742-051X(91)90022-H

Gould, N.y Taylor, I. (2017). Reflective learning for social work: Research, theory and practice. Nueva York: Routledge.

Habermas, J. (1989). The structural transformation of the public sphere: An inquiry into a category of bourgeois society. Cambridge: MIT Press.

Johnston, M. (2005). The lamp and the mirror: Action research and self-studies in the social studies. En K. Barton (ed.), Research methods in social studies education: Contemporary issues and perspectives (pp. 57-83). Greenwich: Information Age.

Kemmis, S.y McTaggart, R. (2005). Participatory action research: Communicative action and the public sphere. En N. K. Denzin y Y. S. Lincoln (eds.), Handbook of qualitative research (3 ed., pp. 559-604). Thousand Oaks: Sage.

Kitchen, J. y Stevens, D. (2005). Self-study in action research: Two teacher educators review their project and practice. Ontario Action Researcher, 8(1). Recuperado de https://www.researchgate.net/publica- 
tion/253479927_SELF-STUDY_IN_ACTION_RESEARCH_TWO_TEACHER_EDUCATORS_REVIEW_THEIR_PROJECT_AND_PRACTICE

Leitch, R. y Day, C. (2000). Action research and reflective practice: Towards a holistic view. Educational Action Research, 8, 179-193. DOI: 10.1080/09650790000200108

McMahon, T. (1999). Is reflective practice synonymous with action research? Educational Action Research, 7, 163169. DOI: 10.1080/09650799900200080

Mann, S. y Walsh, S. (2017). Reflective practice in English language teaching: Research-based principles and practices. Nueva York: Routledge.

Martín-Cabrera, L. (2014). Ciencia, compromiso y cambio social. Textos de Orlando Fals Borda. Revista Latinoamericana, 13(38), 637-641. Recuperado de http://www.redaly c.org/pdf/305/30531773029.pdf

Mata, A., Hernández, P.y Centeno, G. (2018, junio). La práctica reflexiva en los docentes de posgrado: comprender para transformar. Innoeduca. International Journal Of Technology And Educational Innovation, 4(1), 36-43. DOI: 10.24310/innoeduca.2018.v4i1.3594

Miles, M. B.y Huberman, A. M. (1984). Qualitative data analysis: A sourcebook of new methods. Newbury Park: Sage.

Ministerio de Educación de Chile (2008). Marco para la buena enseñanza. Santiago de Chile: Mineduc.

Muñoz, M. y Garay, F. (2018) La investigación como forma de desarrollo profesional docente: Retos y perspectivas. Estudios Pedagógicos, 41(2), 389-399. DOI: 10.4067/S0718-07052015000200023

Nolen, A. L. y Vander Putten, J. (2007). Action research in education: Addressing gaps in ethical principles and practices. Educational Researcher, 36, 401-407. DOI: 10.3102/0013189X07309629

Odhiambo, E. (2010). Classroom research: A tool for preparing pre-service teachers to become reflective practitioners. Journal of Instructional Pedagogies, 4, 1-14. Recuperado de http://www.aabri.com/manuscripts/10482.pdf

Ortiz, M. y Borjas, B. (2008). La investigación-acción participativa: Aporte de Fals Borda a la educación popular. Revista Espacio Abierto, 17, 615-627. Recuperado de https://www.redaly c.org/articulo.oa?id=12217404

Paukner, F. (2005). La reflexión sobre la práctica, una aproximación pedagógica. Boletín de Educación de la Universidad Católica del Norte, 36, 23-42.

Pitsoe, V.y Maila, M. (2013). Re-thinking Teacher Professional Development through Schön's Reflective Practice and Situated Learning Lenses. Mediterranean Journal of Social Sciences 4(3), 1. DOI: 10.5901/mjss.2013. v4n3p211

Paugh, P., Wendell, K. B., Power, C. y Gilbert, M. (2017). It's not that easy to solve: edTPA and preservice teacher learning. Teaching Education, 29(2), 147-164. DOI: 10.1080/10476210.2017.1369025 
Price, J. y Valli, L. (2005). Preservice teachers becoming agents of change: Pedagogical implications for action research. Journal of Teacher Education, 56, 57-72. DOI: 10.1177/0022487104272097

Project Zero y Reggio Children (2001). Making learning visible: Children as individual and group learners. Reggio Emilia, Italia: Reggio Children.

Riel, M. (2017). Understanding action research. Pepperdine University, Center for Collaborative Action Research. http://cadres.pepperdine.edu/ccar/define.html

Ryan, M. (2013). The pedagogical balancing act: teaching reflection in higher education. Teaching in Higher Education, 18(2), 144-155. DOI: 10.1080/13562517.2012.694104

Schön, D. A. (1983). The reflective practitioner. Nueva York: Basic Books.

Schön, D. A. (1987). Educating the reflective practitioner: Toward a new design for teaching and learning in the professions. San Francisco: Jossey Bass.

Strauss, A. y Corbin, J. (1994). Grounded theory methodology: An overview. En N. K. Denzin y Y. S. Lincoln (eds.), Handbook of qualitative research (pp. 1-18). Londres: Sage.

Van Manen, M. (1995). On the epistemology of reflective practice. Teachers and Teaching: Theory and Practice, 1, 33-50. DOI: 10.1080/1354060950010104 
